\title{
Tracking and refocussing of moving targets in multichannel SAR data
}

\author{
Henke, Daniel ; Meier, Erich
}

\begin{abstract}
Moving targets in Synthetic Aperture Radar (SAR) data impose several challenges for SAR processing. In full-aperture SAR images, moving objects appear smeared and/or displaced. In previous studies, we showed that a combination of sub-aperture processing, feature extraction, unscented Kalman filtering and multi-target hypotheses can reliably detect and track moving objects. In this paper, we present some extensions to the tracking algorithm itself, show results on a newly acquired data set and demonstrate how the estimated trajectories of the moving objects can be utilized to refocus the originally smeared objects.
\end{abstract}

DOI: https://doi.org/10.1109/igarss.2015.7326635

Posted at the Zurich Open Repository and Archive, University of Zurich ZORA URL: https://doi.org/10.5167/uzh-116860

Conference or Workshop Item

Published Version

Originally published at:

Henke, Daniel; Meier, Erich (2015). Tracking and refocussing of moving targets in multichannel SAR data. In: Geoscience and Remote Sensing Symposium (IGARSS), 2015 IEEE International, Milan, Italy, 26 July 2015 - 31 July 2015. IEEE, 3735-3738.

DOI: https://doi.org/10.1109/igarss.2015.7326635 


\title{
TRACKING AND REFOCUSSING OF MOVING TARGETS IN MULTICHANNEL SAR DATA
}

\author{
Daniel Henke, Erich Meier \\ Remote Sensing Laboratories (RSL), University of Zurich
}

\begin{abstract}
Moving targets in Synthetic Aperture Radar (SAR) data impose several challenges for SAR processing. In fullaperture SAR images, moving objects appear smeared and/or displaced. In previous studies, we showed that a combination of sub-aperture processing, feature extraction, unscented Kalman filtering and multi-target hypotheses can reliably detect and track moving objects. In this paper, we present some extensions to the tracking algorithm itself, show results on a newly acquired data set and demonstrate how the estimated trajectories of the moving objects can be utilized to refocus the originally smeared objects.
\end{abstract}

\section{INTRODUCTION}

Ground moving target indication (GMTI) in SAR data addresses the task of extracting information about moving objects (e.g. [1], [2]). Compared to other GMTI systems, SAR sensors have the great advantage of detecting moving objects while simultaneously imaging the area of interest. Furthermore, SAR data can be acquired almost independently of weather conditions and daylight. In [3] and [4], we developed a flexible GMTI approach which - compared to traditional SAR/GMTI - is capable of not only indicating the presence of moving targets, but also the extraction of an object's motion while in the beam of the SAR sensor by making use of temporal processing methods. Based on these findings, in this paper, we enhance the method of [4] using improved methods of combining single-channel and alongtrack interferometric data for more robust trajectory estimations. This is realized by including additional features into the tracking model, i.e. size, intensity and phase of an object. We validate the extended method on data acquired with DLR's F-SAR sensor [5] based on two campaigns covering the same test area conducted in the years 2010 and 2013, flown with circular acquisition geometries. Furthermore, a refocusing of detected moving objects based on the previously extracted trajectories is addressed. As the relative velocity between moving object and sensor are known from the tracking algorithm, it can be used to refocus the SAR data with object-specific velocity values leading to a focusing of the object of interest while simultaneously defocusing static objects. This can support the identification of certain properties of the object of interest.

\section{METHOD}

\subsection{Test site and sensor}

The GMTI experiments took place in Härkingen $(\sim 55 \mathrm{~km}$ west of Zurich) and were recorded twice (in 2010 \& 2013) with circular flight geometries (see Fig.1) using DLR's FSAR sensor [5]. Important sensor specifications can be found in the Tab. 1: a higher range bandwidth was used 2013.
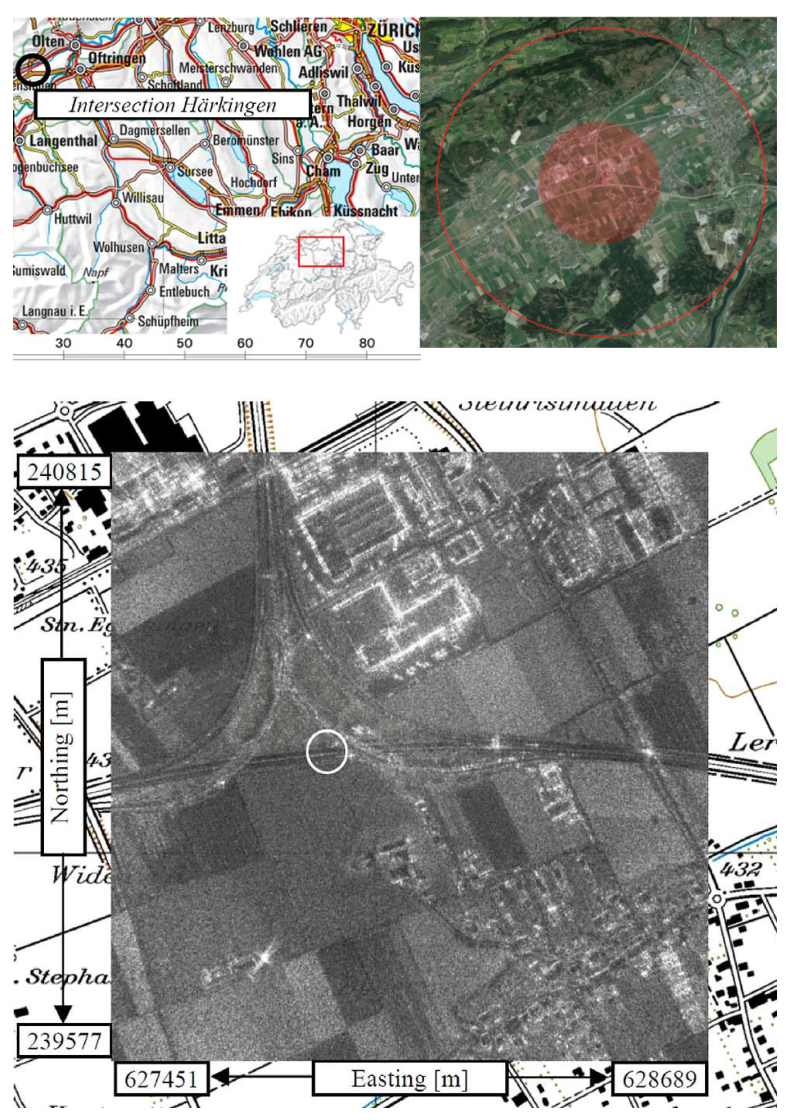

Fig. 1: Top: test site Härkingen; bottom: SAR image overlaid on a topographic map. 


\begin{tabular}{|c|c|c|c|c|c|c|}
\hline $\begin{array}{l}\text { DATA } \\
\text { TAKE }\end{array}$ & BAND & PRF & BANDWIDTH & CHANNEL & HEADING & YEAR \\
\hline HK1 & $\begin{array}{c}\mathrm{X} @ 9.6 \\
\mathrm{GHz}\end{array}$ & $2016 \mathrm{~Hz}$ & $100 \mathrm{MHz}$ & 4x ATI & $0^{\circ}-360^{\circ}$ & 2011 \\
\hline HK2 & $\begin{array}{c}\mathrm{X} @ 9.6 \\
\mathrm{GHz}\end{array}$ & $2404 \mathrm{~Hz}$ & $384 \mathrm{MHz}$ & 4x ATI & $0^{\circ}-360^{\circ}$ & 2013 \\
\hline
\end{tabular}

Tab. 1: Sensor specifications.

\subsection{Extended target tracking algorithm}

In [4], integration methods for a combined single-channel and multi-channel target tracking were based solely on the center of mass. This can lead to gaps in the trajectory of an object, especially in the transitions between ATI-supported and single-channel-supported observations. To overcome that, we use additional features - namely phase $\phi$, intensity $I$ and size $A$ of an object - to merge the two observation sets in a more robust way.

Starting from the model with only the center of mass ([4]), different possibilities of embedding the new combined observation ( $\hat{=}$ feature) set into the tracking model are conceivable. We chose a solution where the size, intensity and phase are included in the state space: $x=\left(\operatorname{pos}_{\text {north }}, \operatorname{pos}_{\text {east }}, v_{a b s}, \varphi, A, I, \phi\right)$. The state transition function $f(x)$ was assumed to show a constant temporal behavior of the newly integrated observation set. Given this definition of the state and state transition function, the transformation function $h(x)$ mapping the state into the observation state can be derived, and the state is updated according to the Kalman gain (for equations, see [4]).

Including combined features from both the along-track interferometry (ATI) and single-channel data not only in the detection step but also in the temporal tracking model, allows a more robust estimation of the trajectories.

\subsection{Refocusing}

Using standard SAR focusing, static objects are well-focused; however, moving objects are smeared and/or displaced. Given the knowledge of the objects' motions obtained by the tracking algorithm, a refocusing of the moving objects becomes possible, providing additional useful information on the object's properties. The quality of the refocused object depends on (a) the accuracy of the estimated motion parameters and (b) on the relationship between object's size and sensor resolution. As a result of the refocusing, the moving object is focused while static targets become smeared and displaced. The refocusing works automatically and is based on the previously calculated set of trajectories: the echo pulses in the time-domain back-projection (TDBP) algorithm are summed up using the offset shifts induced by the estimated object's movement.

\section{RESULTS}

\subsection{Extended target tracking algorithm}

With only the "center of mass"-feature (see [4]) and a certain interval of missing observations at single detection steps, sometimes the track of an object is lost and when observations of the same object are detected again at single time instances later in the process, a new object is created instead of bridging the gap. Two such examples were reported in [4]. With the extended tracking model using information from both data sources (ATI and single-channel), these intervals of missed observations can be linked together. This higher probability of maintaining the track without interruptions in the single-channel/ATI transition can be confirmed for the 2013 data set where no gaps in the tracks occurred. This is caused by not only using the position but also size, intensity and phase. A snapshot of the output of the tracking algorithm as seen from the sensor platform is presented in Fig. 2.

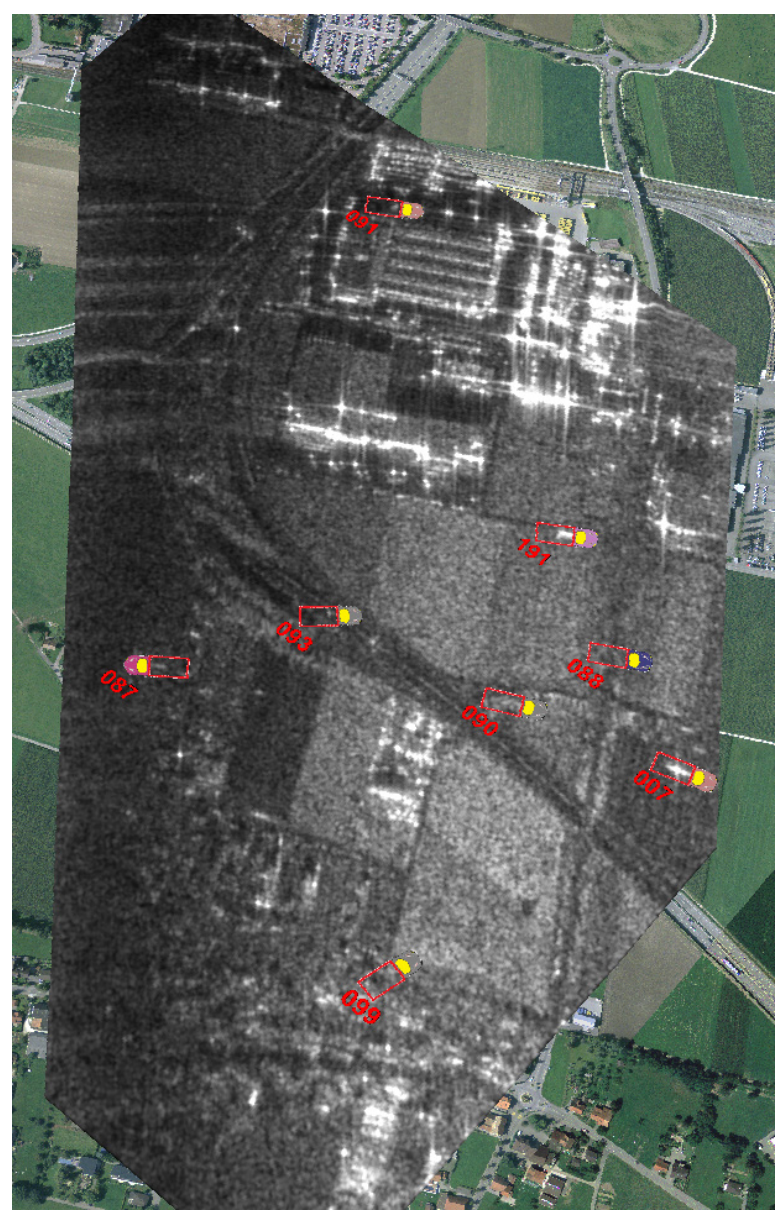

Fig. 2: Snapshot of the tracked objects in test site Härkingen. (Point of view: sensor platform) 


\subsection{Refocusing}

Fig. 3 shows examples of 3 trajectories obtained by the GMTI algorithm and Fig. 4 the corresponding refocused images.

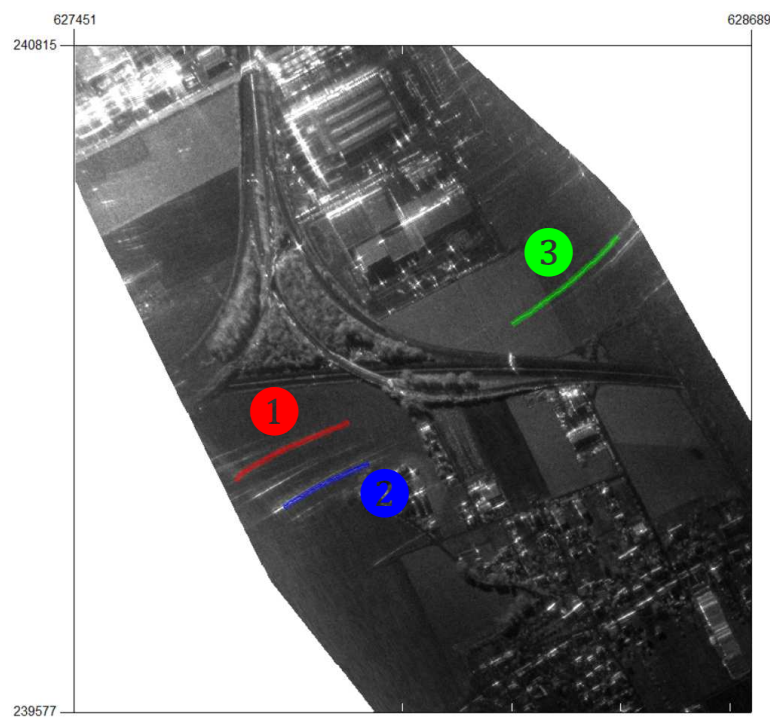

Fig. 3: Trajectories of the GMTI algorithm

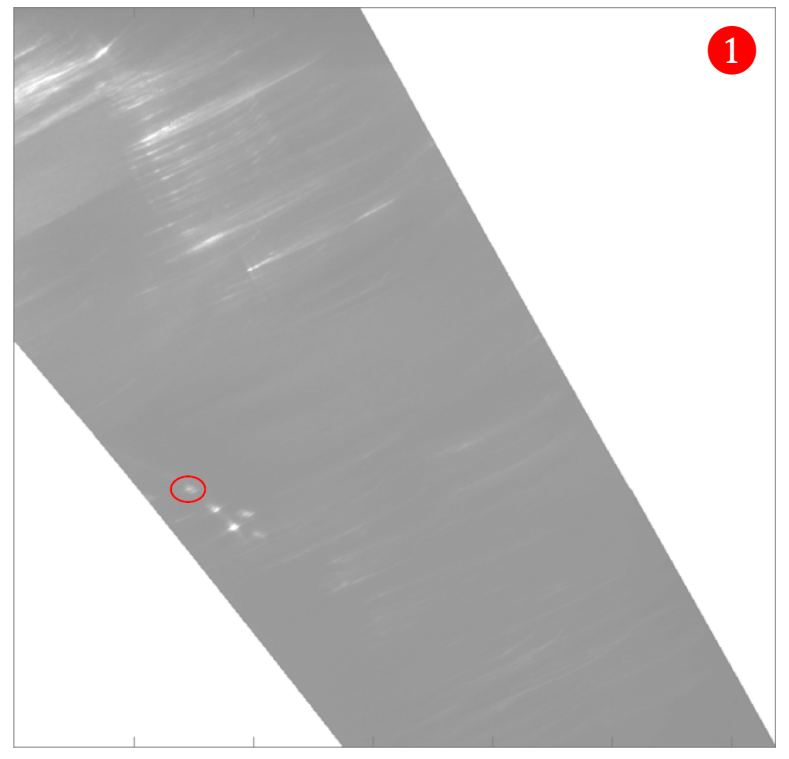

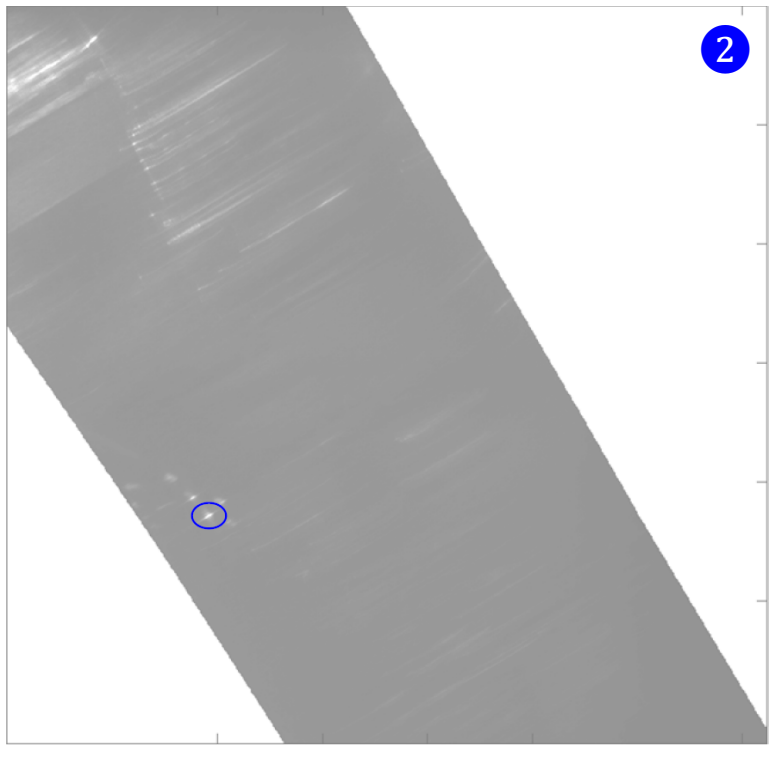

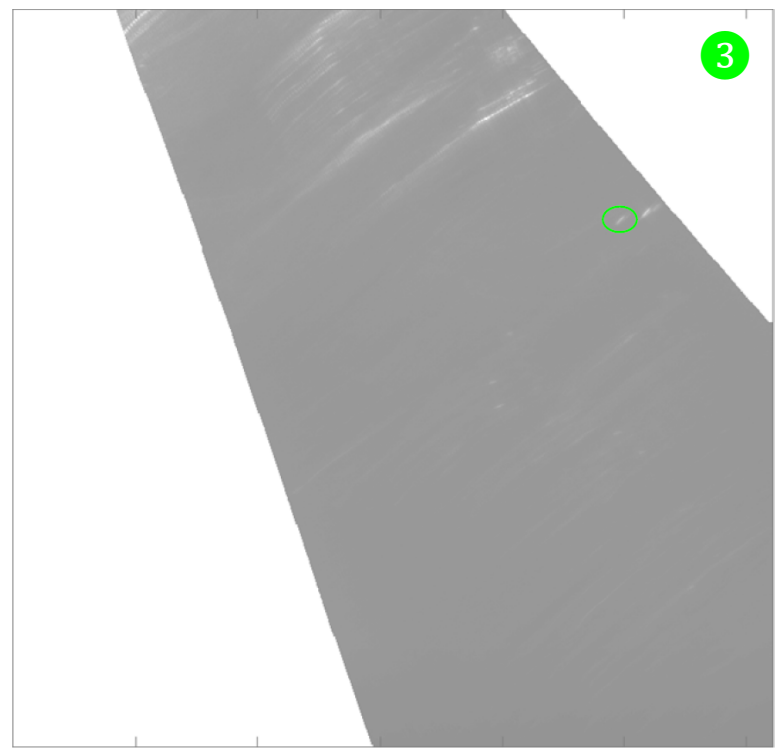

Fig. 4: Images refocused with the motion parameters of the respective objects of Fig. 3.

Fig. 5 presents zoomed versions of the three refocused images of Fig. 4 with the best focusing quality obtained for the blue vehicle (center image). The black line in the images indicates the $3 \mathrm{~dB}$ contour of the objects as a measurement of the objects' dimension. Typical values of the objects' length vary between $10 \mathrm{~m}$ and $20 \mathrm{~m}$; the objects' width is in the order of 3 $\mathrm{m}$. These are realistic dimensions for trucks. However, statistical inferences on the object's characteristics have been difficult to derive up to now as neither the scattering mechanisms of trucks under different illumination angles nor 
the precise radiometric calibration have been investigated in detail.
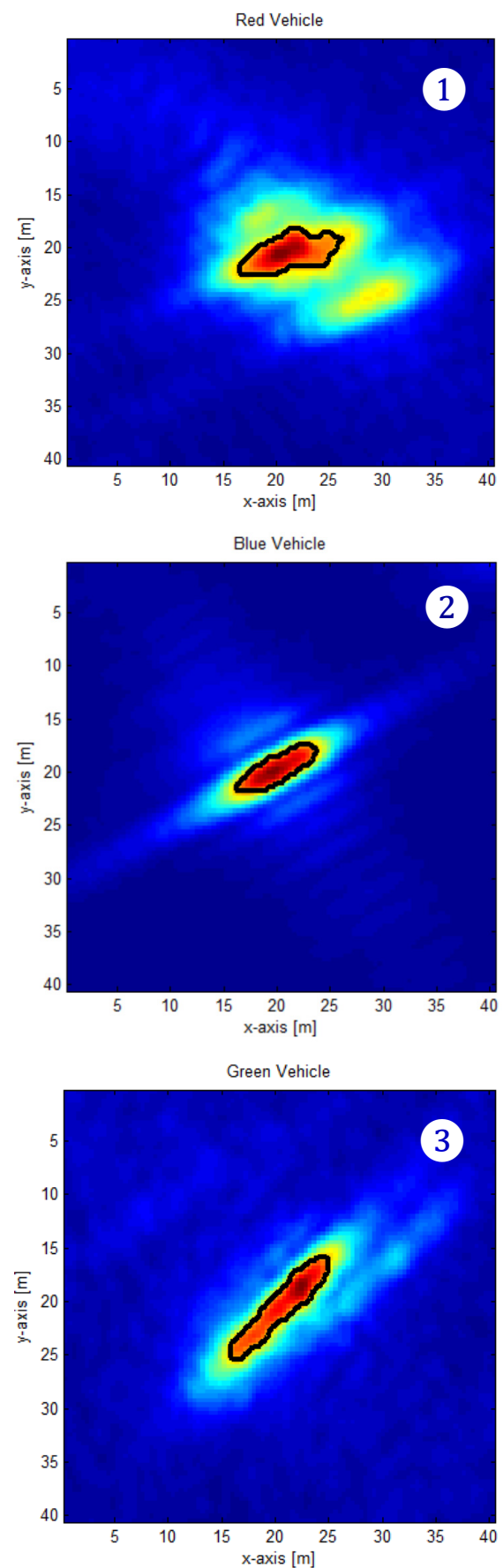

Fig. 5: Refocused version of the three objects corresponding to the images of Fig. 4. The black line indicates the $3 \mathrm{~dB}$ contour of the object.

\section{CONCLUSION}

An extended multichannel GMTI algorithm was presented and validated on two F-SAR data sets. The derived trajectories were used to refocus moving objects providing preliminary information on the dimensions and scattering mechanisms of the object of interest. For an improved interpretation of the refocused objects, the investigation of vehicle signatures will be needed. Furthermore, postprocessing steps such as autofocus techniques optimizing an SNR maximization criterion are potential future research directions to minimize errors caused by small deviations of the estimated trajectories. The tracking algorithm itself and the refocused images of moving objects were demonstrated to be very useful for SAR/GMTI and moving object refocusing applications.

\section{ACKNOWLEDGEMENTS}

This work was supported in part by armasuisse $\mathrm{W}+\mathrm{T}$ and the Forschungskredit of the University of Zurich, grant no. FK14-105.

\section{REFERENCES}

[1] D. Cerutti-Maori, I. Sikaneta, and C. H. Gierull, "Optimum SAR/GMTI processing and its application to the radar satellite RADARSAT-2 for traffic monitoring," IEEE Transactions on Geoscience and Remote Sensing, vol. 50(10), pp. 3868-3881, 2012.

[2] V. T. Vu, T. K. Sjogren, M. I. Pettersson, A. Gustavsson, and L. M. Ulander, "Detection of moving targets by focusing in UWB SAR-Theory and experimental results," IEEE Transactions on Geoscience and Remote Sensing, vol. 48(10), pp. 3799-3815, 2010.

[3] D. Henke, C. Magnard, M. Frioud, D. Small, E. Meier, and M. E. Schaepman, "Moving-Target Tracking in Single-Channel Wide-Beam SAR," IEEE Transactions on Geoscience and Remote Sensing, vol. 50(11), pp. 4735-4747, 2012.

[4] D. Henke, E. Mendez Dominguez, D. Small, M. E. Schaepman, and E. Meier, "Moving Target Tracking in Single- and Multichannel SAR," IEEE Transactions on Geoscience and Remote Sensing, 2014, vol.53(6), pp.3146-3159, 2015.

[5] A. Reigber, M. Jager, J. Fischer, R. Horn, R. Scheiber, P. Prats, and A. Nottensteiner, "System Status and Calibration of the F-SAR Airborne SAR instrument," in IEEE International Geoscience and Remote Sensing Symposium (IGARSS), pp. 1520-1523, 2011. 